\title{
Birth outcomes relative to dietary vitamin D \& calcium intake in obese pregnant women
}

\author{
M. S. Charnley ${ }^{1}$, J. C. Abayomi ${ }^{1}$ and A. Weeks ${ }^{2}$ \\ ${ }^{1}$ Faculty of Education, Community and Leisure, Liverpool John Moores University, IM Marsh Campus, Barkhill Road, \\ Liverpool L17 6BD and Liverpool Women's Hospital, Crown Street, Liverpool L8 7SS, UK
}

For individuals with limited exposure to ultra-violet B, a recommended nutrient intake (RNI) of $10 \mu \mathrm{g} /$ day of vitamin D is recommended; this includes all pregnant and lactating women ${ }^{(1)}$. Despite this, research suggests a subset of pregnant women are at risk of vitamin D insufficiency due to obesity, darker skin pigmentation and estimated delivery date in spring or summer ${ }^{(2)}$. There is no increase in requirements for calcium during pregnancy however a positive maternal calcium balance is dependent on adequate circulating levels of $25(\mathrm{OH}) \mathrm{D}_{3}{ }^{(3)}$. Maternal outcomes such as gestational diabetes, pre-eclampsia and increased risk of caesarean section are all associated with low vitamin D status ${ }^{(4)}$. The aim of this study was to investigate whether dietary intakes of vitamin D and calcium were associated with adverse maternal and birth outcomes. Participants were asked to complete three-day food diaries during each trimester of pregnancy. Data regarding food portion size was verified using a food atlas ${ }^{(5)}$ and the diaries were then analysed using Microdiet ${ }^{(i m}$.

Table 1. Mean Vitamin D and calcium intake over 3 trimesters

\begin{tabular}{|c|c|c|c|c|c|c|c|c|c|}
\hline & \multicolumn{2}{|c|}{ Total energy } & \multicolumn{5}{|c|}{ Vitamin D } & \multicolumn{2}{|c|}{ Calcium } \\
\hline & Mean Kcals/d & SD & Mean $\mu \mathrm{g} / \mathrm{d}$ & SD & RNI \% $10 \mu \mathrm{g} / \mathrm{d}$ & Mean mg/d & SD & RNI \% 700mg/d & LRNI \% 400mg/d \\
\hline Visit 1 16-20wks $(n=93)$ & 1849 & 590.7 & 2.6 & 2.5 & 26 & 875 & 334.4 & 125 & 219 \\
\hline Visit 2 28wks $(n=99)$ & 1984 & 525.6 & 2.4 & 2 & 24 & 949 & 356.8 & 136 & 237 \\
\hline Visit 3 36wks $(n=73)$ & 2066 & 587.2 & 2.74 & 2.5 & 27 & 994 & 423.6 & 144 & 252 \\
\hline
\end{tabular}

Data were collected for 140 women with a BMI $\geq 35 \mathrm{~kg} / \mathrm{m}^{2}(n=139)$, a mean booking-in weight of $110 \mathrm{~kg}$ (SD 15.5$)$ and mean birth weight of $3.57 \mathrm{~kg}$ (SD 0.67). Most women achieved total energy requirements at all 3 visits however there was a wide distribution around the mean with an average minimum intake of $706 \mathrm{kcals}$ for all 3 visits and a maximum intake of $3906 \mathrm{kcals}$. There was a positive dietary intake for calcium with $73.6 \%$ of women achieving RNI and $95.7 \%$ achieving LRNI. Dietary intake of vitamin D was low with only $2.2 \%$ of women achieving RNI at all 3 visits. Spearman's correlation suggests an association with vitamin D and birth weight (rho $=0.224$, $p=0.036$ ) at visit 1 . This suggests that vitamin D status in early pregnancy may influence birth weight and that pre-natal supplements may be necessary. Results indicate that further investigation into the quality of maternal diet and pregnancy outcomes is required.

1. COMA (1991) Dietary Reference Values for Food, Energy \& Nutrients for the UK. HMSO: London.

2. Dror DK \& Allen LH (2010) Vitamin D inadequacy in pregnancy: biology, outcomes, and interventions Nutrition Review 68(8): 465-477.

3. Philip W \& James T (2008) 22nd Marabou Symposium: the changing faces of vitamin D Nutrition Reviews 66, S213-S217.

4. Hollis BW \& Wagner CL (2006) Nutritional vitamin D status during pregnancy: reasons for concern CMAJ 174(9): 1287-1290.

5. Nelson M, Atkinson M \& Meyer J (2002) A photographic atlas of food portion sizes FSA. 